\title{
STEM-EDS Characterization of Platinum-Modified Nickel Nanoparticles
}

\author{
Dalaver H. Anjum ${ }^{1}$ and Lidong $\mathrm{Li}^{2}$
}

1. Imaging and Characterization Lab, King Abdullah University of Science \& Technology (KAUST), Thuwal, Kingdom of Saudi Arabia (KSA)

2. KAUST Catalysis Center (KCC), KAUST, Thuwal, KSA

Nickel nanoparticles (Ni NPs) are quite interesting because they exhibit high catalytic activity, high selectivity and high efficiency in fuel cells $[1,2]$. However the coke-formation takes place in these NPs involving chemical reactions. For instance the $\mathrm{CO}_{2}$ reforming of methane gas in the presence of Ni NPs leads to the formation of coke containing predominantly the carbon nanotubes (CNTs) [3]. Coke formation is detrimental to the efficiency of chemical reactions and hence results in the deactivation of catalysts and blockage of tubular reactor. This translates to higher amount of energy usage which is both costly as well as is not conducive to environment. Surface modification of Ni NPs with noble-metals not only reduces such coke formation but it also enhances their catalytic activity. In regards to the synthesis of bimetallic NPs, the approach of using surface organometallic chemistry (SOMC) method is both versatile as well as low-cost. However the success of SOMC method to produce uniform size and composition bimetallic NPs can be judged only after their successful characterization with an elaborate technique. Moreover the real space imaging and characterization of NPs is vital to understanding their structure-property relationships and transmission electron microscopy (TEM) helps enormously in the determination of such relationships [4].

This report contains a study that involves the characterization of pure Ni and Pt-modified Ni NPs. It has been carried out by using the technique of scanning TEM (STEM) in conjunction with energy dispersive spectroscopy (EDS). The both type of samples were loaded onto

$\gamma-\mathrm{Al}_{2} \mathrm{O}_{3}$ support and their catalytic activity was thus measured subsequently by the $\mathrm{CO} 2$ reforming with methane gas for the duration of twenty-four hours. All the samples before and after the reaction (4 in total) were then analyzed by employing FEI Company's Titan $\mathrm{G}^{2}$ 60-300 ST TEM which was equipped with a Cs-corrector for the probe from CEOS and also with an EDS detector from EDAX.

A STEM micrograph, acquired by using an high-angle annular dark-field (HAADF) detector, of Ni/ $\gamma-\mathrm{Al}_{2} \mathrm{O}_{3}$ sample before reaction (BR) is shown in Figure 1 (A) and it contains bright-color NPs along with gray-color NPs. The average size of bright-color NPs was found out to be in the range of $6.5+/-$ $1.5 \mathrm{~nm}$. Owing to the presence of Z-contrast in the HAADF-STEM micrographs, it can be inferred that the bright-color NPs are of Ni metal and the gray-color NPs are of $\gamma-\mathrm{Al}_{2} \mathrm{O}_{3}$ support. The presence of $\mathrm{Ni}$ NPs was confirmed further by a corresponding EDS spectrum which is shown as an insert in the upper-right corner of the Figure 1 (A). Similarly Figure 1 (B) also contains a HAADF-STEM micrograph from the same Ni NP sample but after reaction (AR). The presence of CNTs can be readily noticed in the micrograph which is indicating the formation of coke after the $\mathrm{CO} 2$ reforming with methane gas. The ripening of Ni NPs also took place as their average size increased to about $11+/-3$ $\mathrm{nm}$. However, the structure of these NPs remain crystalline after the ripening as it can be confirmed by presence of lattice-fringes in a highresolution STEM (HR-STEM) micrograph shown as an insert in the upper-right corner of Figure 1 (B). 
An HAADF-STEM micrograph of Pt-modified Ni NPs sample before reaction (BR) is shown in Figure 1 (C) that presumably contain Pt-modified Ni NPs, whose size is found out to be in the range of about $3.5+/-1.5 \mathrm{~nm}$, along with $\gamma-\mathrm{Al}_{2} \mathrm{O}_{3}$ support. Again, the EDS analysis that is shown as an insert in upper-left corner of the Figure 1 (C) confirmed the presence of Pt metal in this sample. The surface decoration of Ni NPs with Pt atoms was investigated with their HR-STEM analysis and it is also shown as an inset in the lower-right corner of Figure 1(C). Owing to the Z-contrast in DF-STEM, it seems that the Pt metal tend to decorate the surface of Ni NPs both in the form of atom-by-atom as well as clusters. However these bimetallic NPs sinter to larger size NPs $(\sim 4.5+/-2 \mathrm{~nm})$ after reaction (AR) as shown by an HAADF-STEM micrograph of Figure 1 (D). This micrograph further shows that the irregular shape Pt-modified Ni NPs change to quite regular spherical shape NPs after reaction but no coke formation was observed in this sample. It is an evidence of showing a superior catalytic performance of these Pt-modified Ni NPs as compared to pure Ni NPs. In summary it can be stated that the surface modification of Ni NPs with Pt metal by using SOMC method is possible. Finally the STEM-EDS analysis of such type of bimetallic NPs allows investigating their structure-property relationships quite successfully.

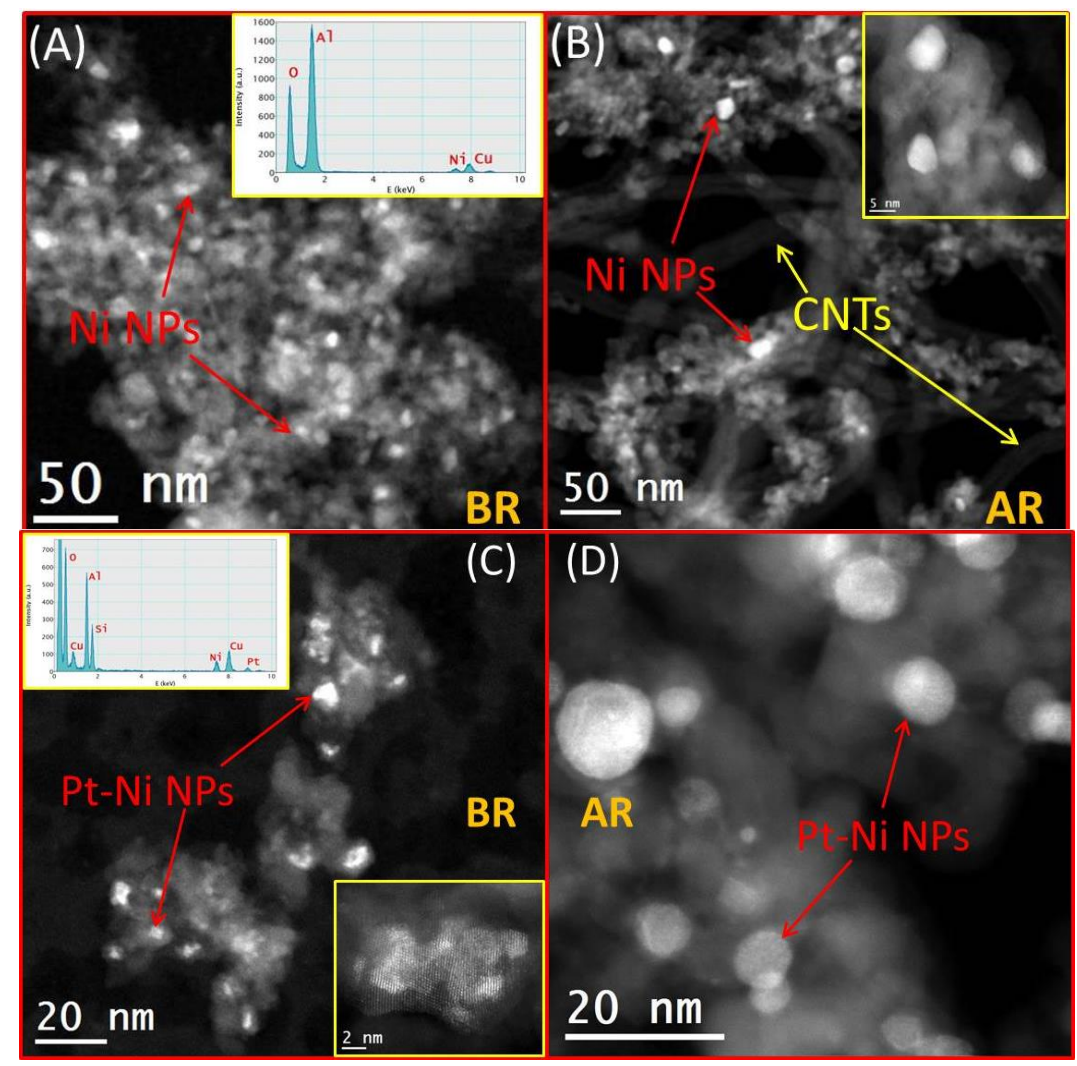

Figure 1: HAADF STEM-EDS analysis of Pt-modified Ni NPs loaded onto $\gamma-\mathrm{Al}_{2} \mathrm{O}_{3}$

\section{References:}

[1] Y. Du et al., Appl. Catal., A, 277, 259-264 (2004).

[2] W. Wang et al., Chem. Rev., 113, 8104-8151 (2013).

[3] C. J. Liu et al., ChemCatChem, 3, 529-541 (2011).

[4] P. Buseck, J. M. Cowley, and L. Eyring eds, High Resolution Transmission Microscopy and

Associated Techniques, New York, London, Amsterdam, Oxford University Press, (1988). 